\title{
High-throughput scanning electrochemical microscopy brushing of strongly tilted and curved surfaces
}

\author{
Andreas Lesch ${ }^{\mathrm{a}, \mathrm{b}, 2}$, Dmitry Momotenko ${ }^{\mathrm{b}}$, Fernando Cortés-Salazar ${ }^{\mathrm{b}, 1}$, Folkert Roelfs ${ }^{\mathrm{a}}$, \\ Hubert H. Girault ${ }^{\mathrm{b}, *, 1}$, Gunther Wittstock ${ }^{\mathrm{a}, * *, 1}$

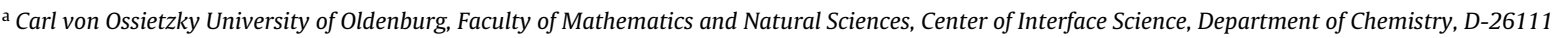 \\ Oldenburg, Germany \\ ${ }^{\mathrm{b}}$ Ecole Polytechnique Fédérale de Lausanne, Laboratoire d'Electrochimie Physique et Analytique, Station 6, CH-1015 Lausanne, Switzerland
}

\section{A R T I C L E I N F O}

\section{Article history:}

Received 10 December 2012

Received in revised form 12 March 2013

Accepted 14 March 2013

Available online 26 March 2013

\section{Keywords:}

Soft linear microelectrode arrays

Scanning electrochemical microscopy

Contact mode scanning

Strongly tilted substrates

Strongly curved substrates

\begin{abstract}
A B S T R A C T
The feasibility of high-throughput scanning electrochemical microscopy (SECM) of strongly tilted (tilt angles $\leq 4^{\circ}$ ) and curved substrates (diameter of curvature $\geq 9 \mathrm{~cm}$ ) is demonstrated by brushing them with a soft linear array of carbon microelectrodes. This probe made of thin polymeric layers operates in contact regime to follow the topography of highly unconventional SECM samples while keeping an almost constant working distance. Strong slope variations of the sample lead to a slight misalignment between the axes of the positioning system and the sliding direction of the microelectrode arrays. The resulting positional offsets can be predicted and corrected to yield a correct representation of the spatial relation on the surface of the sample. Moreover, a custom-made holder system ideally suited for precise control of the soft probe inclination angle and alignment with the substrate plane was also developed to perform high-throughput SECM imaging of a $1.2 \mathrm{~cm}^{2}$ curved metallic pin within less than $2 \mathrm{~h}$.
\end{abstract}

(c) 2013 Elsevier Ltd. All rights reserved.

\section{Introduction}

Scanning electrochemical microscopy (SECM) is a scanning probe technique that is conventionally carried out with an amperometric disc-shaped ultramicroelectrode (UME; typically denoted as "tip") localized in the vicinities of a sample substrate and in presence of an electrolyte solution [1-4]. Redox-active species detected or generated at the UME and/or sample are employed to determine local surface reactivity or to manipulate interfaces with micro- and submicrometer resolution [5-11].

The recorded amperometric signals at the microelectrode rely on the diffusional mass transport of the present redox-active species and the local surface reactivity of the substrate under study. Typically, SECM imaging is performed by lateral probe scanning in constant height mode, since variations in the probe-sample distance $(d)$ strongly affect the SECM response and performance. Typically, the loss of lateral resolution and a decrease of current

\footnotetext{
* Corresponding author. Tel.: +41 21693 3151; fax: +41 216933667 .

** Corresponding author. Tel.: +49 441798 3971; fax: +49 4417983979.

E-mail addresses: andreas.lesch@epfl.ch (A. Lesch),dmitry.momotenko@epfl.ch (D. Momotenko), fernando.cortessalazar@epfl.ch (F. Cortés-Salazar),

folkert.roelfs@uni-oldenburg.de (F. Roelfs), hubert.girault@epfl.ch (H.H. Girault), gunther.wittstock@uni-oldenburg.de (G. Wittstock).

1 ISE member.

2 ISE member from January 2013.
}

contrast are observed between reactive and inert surface regions when increasing $d$. The possibility for a mechanical probe-sample contact poses the risk of a very undesired "tip crash" event when decreasing $d$. In order to avoid this situation, many efforts have been made to level the sample and the horizontal scanning plane of the UME, which is particularly important and difficult for the investigation of large samples such as latent human finger prints [12,13], for the screening of combinatorial material libraries [14-16], and for the testing of the integrity of technical coatings [17-19]. Furthermore, sample tilt elimination becomes impossible for curved and corrugated substrates. Several approaches have been proposed to incorporate additional probe-distance control mechanisms based on the measurements of the negative feedback current [20], impedance $[21,22]$ or alternating current signals [23]. The usage of dual UME probes, in which one UME detects the species under investigation whereas the second one uses an independent reaction (e.g. the oxygen reduction) for determining the working distance was also reported [20]. An alternative route is the combination of SECM with other scanning probe techniques that can control the probe-sample distance, such as scanning force microscopy (SFM) [24-30], electrochemical scanning tunnelling microscopy (ECSTM) [31,32], and scanning ion-conductance microscopy (SICM) [33,34]. Other SECM hybrid techniques for distance control rely on the reduced damping of a vertically or laterally vibrated probe in close proximity to the substrate. They include the tip-position modulation (TPM) [35-37], shear-force detection 
[38-46], intermittent-contact mode (IC) [47] and voltage switching mode (VSM) [48]. However, most of these approaches require additional instrumentation and/or are limited to the use of nanoelectrodes that demands a significantly smaller $d$ [44]. This raises at the same time the potential for a probe-sample crash when scanning fast over long distances, and often, there is no need for nanometre resolution in SECM imaging of large sample areas $\left(\mathrm{mm}^{2}\right.$ to $\mathrm{cm}^{2}$ ).

Recently, we have introduced soft carbon and gold microelectrode probes that are made in thin polymeric materials providing flexibility and mechanical stability to SECM imaging [49,50]. These probes are scanned in contact mode over surfaces in a brushinglike way achieving an almost constant $d$. This enables scanning of 3D patterns [51], slightly tilted [51] or even vertically orientated substrates [52] without additional instrumentation for distance control. Even initially dry surfaces can be scanned if the mediator solution is provided and removed by means of microfluidics integrated into the probe [52]. Delicate surfaces such as self-assembled monolayers have been brushed without damaging them due to a very small mechanical pressure exerted by the probes on the surface $[49,53]$. Since the amperometric current response in SECM relies on the diffusional mass transport of the redox-active species with a time constant $\tau \approx r_{\mathrm{T}}^{2} \cdot D^{-1}$, where $r_{\mathrm{T}}$ is the radius of the UME and $D$ is the diffusion coefficient of the redox species, probe translation rates faster than $25 \mu \mathrm{m} \cdot \mathrm{s}^{-1}$ have to be avoided in order to achieve an appropriate resolution and to exclude convectional disturbances [54]. This results in imaging times of more than $48 \mathrm{~h}$ for square centimetre-sized areas when using single UMEs and introduce inconveniences when imaging samples that may change during the experiment (e.g. biological samples). Likewise this is not in line with high-throughput approaches (e.g. industrial applications and quality testing). For this purpose, we have recently presented different soft linear arrays of microelectrodes as SECM probes $[49,50,53,55]$. Earlier approaches for SECM multi-tips were also reported, such as the two-dimensional imaging of $\mathrm{O}_{2}, \mathrm{H}_{2} \mathrm{O}_{2}$ and glucose distributions by an array of 400 individually addressable Pt microelectrodes by Meyer et al. [56], the linear independent 16 Pt microelectrode array from Barker et al. [57] and the Au multinanotip electrode from Deiss et al. [58]; however, in contrast to the soft probe arrays, the earlier developed approaches do not combine a constant working distance mode with individually addressable sensors for high-throughput reactivity imaging and surface modification.

Herein, we report the application of soft linear microelectrode arrays for scanning strongly tilted and curved substrates. A probesample alignment is achieved by the usage of a new probe holder and an electronic tilt table. However, strong changes in surface topography cause a difference between the step size in the horizontal plane of the positioning system and the step size on the surface of the tilted or corrugated sample. We provide a quantitative description of these effects followed by a discussion of the importance for the experimental applications of various sample shapes and the significance in data interpretation. Sample tilt angles of up to $4^{\circ}$ are corrected during off-line image processing and represent extreme cases with respect to common laboratorial practise (vide infra).

\section{Experimental}

\subsection{Chemicals}

Ferrocenyl methanol (FcMeOH, $\geq 97 \%$, Sigma-Aldrich, Buchs, Switzerland, and Alfa Aesar, Karlsruhe, Germany), $\mathrm{KNO}_{3}(\geq 99 \%$, Sigma-Aldrich, Buchs, Switzerland, or Carl Roth, Karlsruhe, Germany) and $\mathrm{Na}_{2} \mathrm{SO}_{4}$ (99\%, Merck, Darmstadt, Germany) were used as received and were of analytical grade. Deionised water was produced by a Purelab ${ }^{\circledR}$ Classic (Elga LabWater, United Kingdom) and a Milli-Q plus 185 model (Millipore, Zug, Switzerland). For the preparation of carbon microelectrode arrays, $100 \mu \mathrm{m}$ thick foils of polyethylene terephthalate (PET; Melinex Dupont, Wilmington, DE, United States of America), $125 \mu \mathrm{m}$ thick foils of Kapton $\mathrm{HN}^{\circledR}$ polyimide (PI, Goodfellow, Huntingdon, England) and Electrador carbon ink (Electra Polymer \& Chemicals Ltd., Roughway Mill, Dunk Green, England) were used.

\subsection{Preparation of soft microelectrode arrays}

Soft linear arrays of carbon microelectrodes were prepared as described previously [50,51,53]. In brief, microchannels (midpointto-midpoint distance $500 \mu \mathrm{m}$ ) were UV laser-ablated (193 nm ArF excimer laser; fluence $=250-350 \mathrm{~mJ}$; frequency $=50 \mathrm{~Hz}$; Lambda Physik, Göttingen, Germany) through a metallic mask into the PET film and manually filled with the carbon ink. The average length, width and depth of the prepared microchannels were $6 \mathrm{~cm}, 30 \mu \mathrm{m}$ and $20 \mu \mathrm{m}$, respectively. After curing for one hour at $80^{\circ} \mathrm{C}$, the conductive carbon tracks were covered and sealed with a thin Parylene $C$ coating of 3-5 $\mu \mathrm{m}$ thickness. The cross-section was exposed either by UV laser ablation or by razor blade cutting and defines sickle-shaped active electrode areas $[50,51,53]$. The quality of the electrodes and the shape of the exposed area were inspected using a laser scanning microscope Keyence VK 8700 (Keyence, Osaka, Japan).

\subsection{Preparation of platinum, gold and silver electrodes}

\subsubsection{Gold electrodes}

Glass slides were treated with piranha solution and cleaned by sequential sonication in ethanol, acetone, and purified water followed by drying under an Ar stream. Caution: This mixture reacts violently with all organic material. Piranha solution has to be handled with extreme care to avoid personnel injury and property damage. $100 \mathrm{~nm}$ thick Au layers were deposited in an Edwards Auto 306 vacuum coater (Edwards, Crawley, England) at a pressure less than $5 \times 10^{-6}$ mbar. The film growth was initiated by the thermal evaporation of a $1 \mathrm{~nm}$ thick $\mathrm{Cr}$ (99.99\%, Oerlikon Balzers, Liechtenstein) layer at $<0.1 \mathrm{~nm} \mathrm{~s}^{-1}$ as adhesion promoter. Au (99.99\%, Oerlikon Balzers, Liechtenstein) was subsequently evaporated at $<0.1 \mathrm{~nm} \mathrm{~s}^{-1}$ up to $5 \mathrm{~nm}$, before increasing the deposition rate to $0.2-0.3 \mathrm{~nm} \mathrm{~s}^{-1}$ for the deposition of a $100 \mathrm{~nm}$ layer.

\subsubsection{Platinum electrodes}

Pt electrodes on a glass chip were prepared by standard photolithographic processes. The width, thickness and distances of the Pt bands were $100 \mu \mathrm{m}, 100 \mathrm{~nm}$ and $200 \mu \mathrm{m}$, respectively.

\subsubsection{Silver electrodes}

A homogeneous Ag layer of $100 \mathrm{~nm}$ thickness was deposited on a $125 \mu \mathrm{m}$ thick foil of Kapton $\mathrm{HN}^{\circledR}$ polyimide (PI, Goodfellow, Huntingdon, England) using physical vapour deposition (Leybold Optics LAB 600H evaporator, Alzenau, Germany). Parallel Ag tracks (93 $\mu \mathrm{m}$ width $\times 2 \mathrm{~cm}$ length and $200 \mu \mathrm{m}$ periodicity) were achieved by removing Ag by UV laser ablation (193 nm ArF excimer laser, fluence $=250 \mathrm{~mJ}$, frequency $=50 \mathrm{~Hz}$; Lambda Physik, Göttingen, Germany) through a metallic mask. In addition, the PI was engraved about $2.1 \mu \mathrm{m}$, which resulted in small 3D patterns. The substrate was glued with a two component epoxy resin glue Araldite ${ }^{\circledR}$ (Huntsman Corporation, Salt Lake City, UT, United States of America) on the outside of a plastic cylinder (diameter $\approx 9 \mathrm{~cm}$ ) in a way that the Ag lines were parallel to the symmetry axis of the cylinder. 


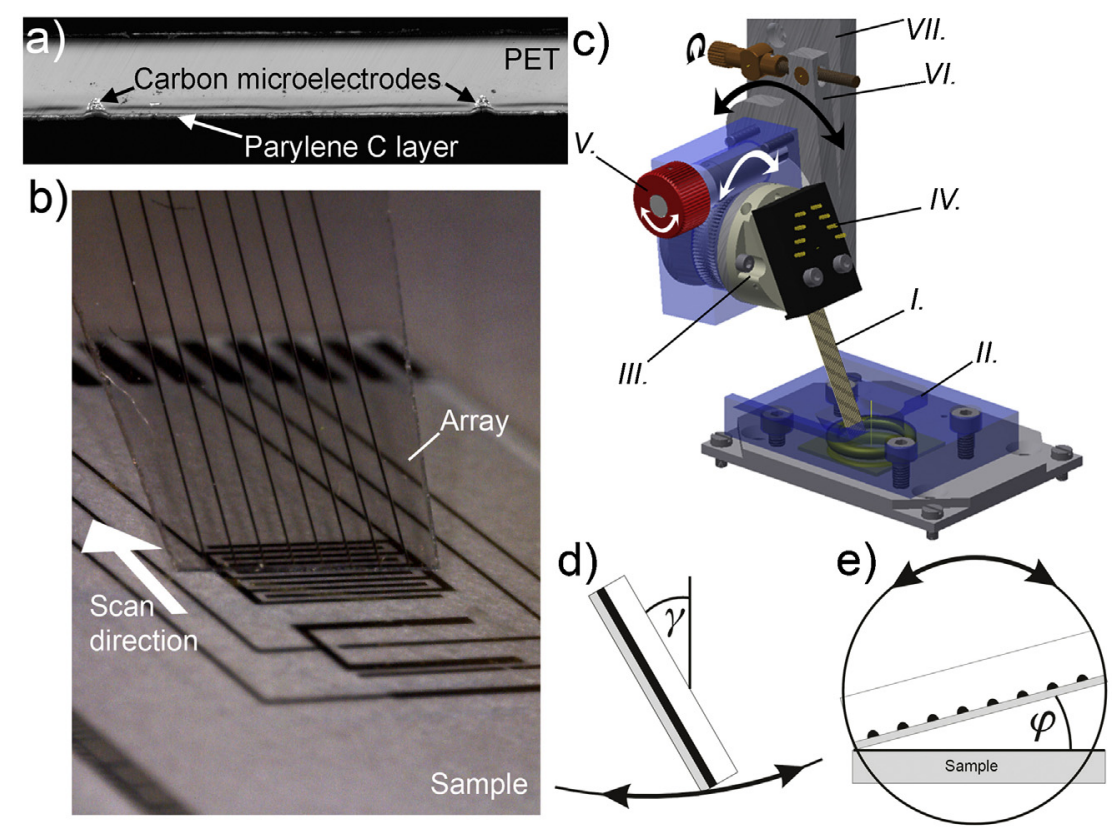

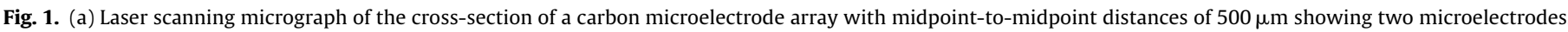

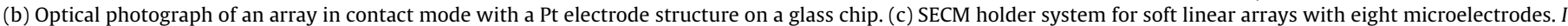

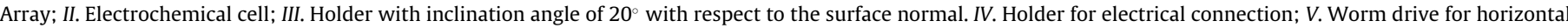

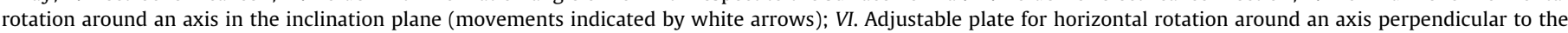
inclination plane (black arrows); VII. Plate to mount at $x, y, z$-positioning system. (d) Schematic of VI. in (c). (e) Schematic of $V$. in (c).

\subsubsection{Test-pin}

A steel pin from the Carl von Ossietzky University of Oldenburg was used as test sample with a corrugated shape. The logo consisting of letters of different fonts had been engraved with different depths (30-60 $\mu \mathrm{m}$ ) and the pin was used as received. It was mounted at the bottom of an SECM cell without tilt correction. Its height profile was measured with a Dektak 150 (Veeco Instruments, Mannheim, Germany).

\subsection{SECM measurements}

All SECM measurements were carried out using a custom-built SECM setup comprising a Märzhäuser three axes positioning system (Märzhäuser GmbH \& Co. KG, Wetzlar, Germany), an electronic tilt table (Zaber Technologies Inc., Vancouver, Canada) and an Ivium multipotentiostat module MultiWE32 connected to an Ivium CompactStat (both Ivium Technologies, Eindhoven, The Netherlands). Up to 32 working electrodes can be controlled individually $v s$. one reference electrode (RE) and one auxiliary electrode (AX) using the in-house made software SECMx [53]. A Pt wire was used as $\mathrm{AX}$ and an $\mathrm{Ag}$ wire served as quasi-RE to which all potentials given herein are referred to. $\mathrm{FcMeOH}$ was used as redox mediator in all experiments and the probe potential $E_{\mathrm{T}}$ was selected between 0.3 and $0.4 \mathrm{~V}$ to achieve a diffusion-limited FcMeOH oxidation at the microelectrodes. The array probe was mounted in a custom-made holder, which will be described in detail in Section 3.1. With this holder, the individual microelectrodes in one array probe can be aligned with the sample surface by taking advantage of a worm drive (vide infra). All samples were used unbiased during the experiments and were fixed at the bottom of the electrochemical cell. The lateral scan direction for reactivity imaging was in $x$-direction, whereas incremental steps in-between line scans were made in $y$-direction. Due to the frequency of probe movement in the horizontal space directions, the $x$-axis is denoted as "high frequency" (HF) axis and the $y$-axis is the "low frequency" (LF) axis.
The sample tilt was controlled by using the electronic tilt table. First, the tilt angles $\delta_{x}$ and $\delta_{y}$ with respect to the horizontal scanning plane of the positioning system (in $x$ - and $y$-direction) were eliminated $\left(\delta=0^{\circ}\right)$ using the three-point approach curve procedure. For this purpose, three approach curves were subsequently performed in a triangular lateral order and $\delta$ were calculated by using the vertical and horizontal distances. $\delta$ were defined as positive when the sample slope was positive with respect to the corresponding horizontal axis. Various $\delta$ of up to $\pm 4^{\circ}$ were used to determine the effect of probe sliding on tilted surfaces.

For SECM line scans and imaging in contact mode, the Parylene $\mathrm{C}$-coated side was facing the sample. Herein, the probe-sample contact is represented by the previously described quantity $h_{\mathrm{P}}$ [49-51,53]. In short, if the probe is placed in solution bulk $h_{\mathrm{P}}$ describes the vertical distance of the probe apex to the sample surface. $h_{\mathrm{P}}$ becomes zero when the probe touches the substrate and negative $h_{\mathrm{P}}$ values describe the probe-apex to sample separation when the probe would penetrate the sample instead of bending and sliding.

SECM imaging was performed using the automatic lift-off (LO) routine, which retracts the soft probes in-between contact mode line scans for repositioning and incremental steps in $y$-direction $[49,53]$. Due to slight variations in sizes, geometries and working distances of the individual microelectrodes, their individual currents vary slightly and were corrected by applying previously described calibration routines $[49,53]$ using the home-made software MIRA. These procedures transform the measured feedback currents $i_{\mathrm{T}, k}$ of the individual microelectrodes $k$ by applying current offsets $i_{\mathrm{T}, \mathrm{offs}, k}$ and scale factors $s_{k}$ in such a way that a dimensionless calibrated current $i_{T, k}^{\prime} / i^{\prime}{ }_{T, \max , k}$ is obtained that becomes zero for the minimum measured current and unity for the maximum measured current.

Calculations to correct the laterally shifted $x$-positions of the individual microelectrode arrays and plotting of the modified SECM image were performed using the software Wolfram Mathematica 8 (Wolfram Research, Champaign, IL, USA). 

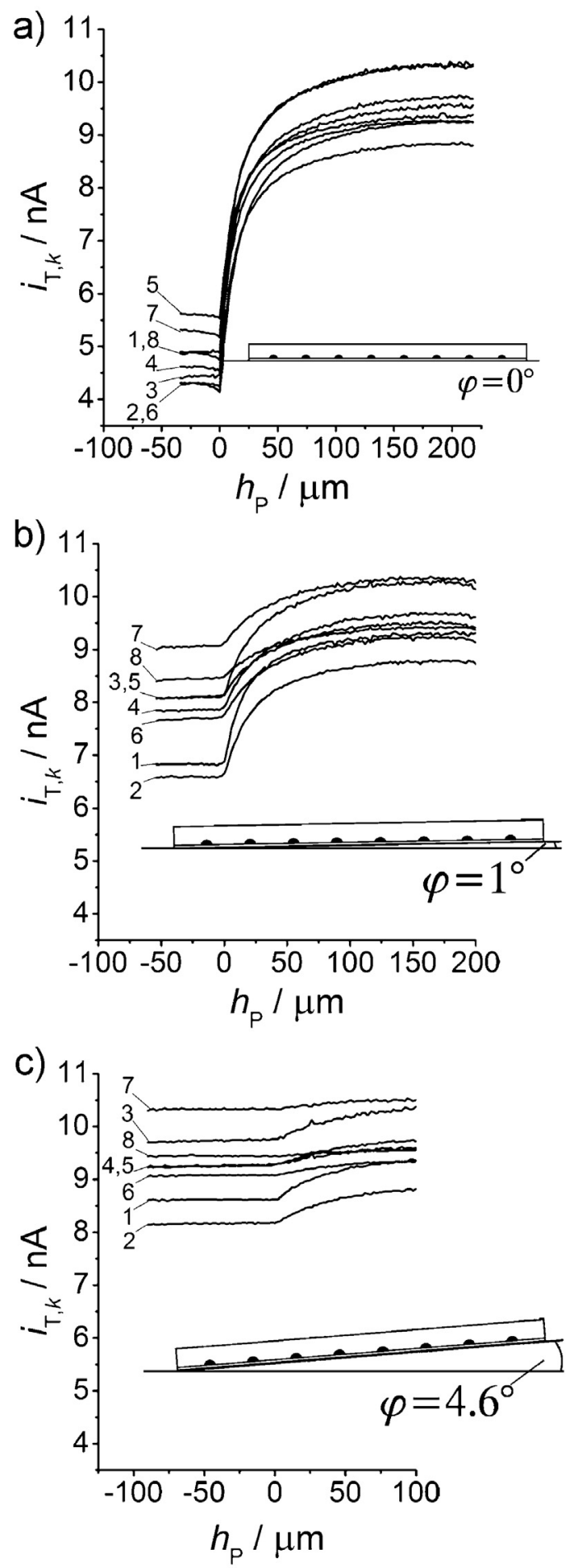

Fig. 2. SECM feedback mode approach curves over insulating glass with a carbon microelectrode array of eight electrodes demonstrating the influence of probe-tosample alignment. Electrolyte solution $2 \mathrm{mM} \mathrm{FcMeOH}$ and $0.1 \mathrm{M} \mathrm{KNO}_{3}$. Step size $2 \mu \mathrm{m}$ and translation rate $5 \mu \mathrm{m} \mathrm{s}^{-1}$. Estimated tilt angles $\varphi$ are $0^{\circ}(\mathrm{a}), 1^{\circ}$ (b) and $4.6^{\circ}$ (c).

\section{Results and discussion}

\subsection{Array-sample alignment}

Typically, the midpoint-to-midpoint distance of the microelectrodes within one soft array is $500 \mu \mathrm{m}$ as shown in the optical micrograph in Fig. 1a. This distance insures that the overlap of the diffusion layers of neighbouring electrodes is negligible [50,59]. For reactivity imaging and surface modification, the probe is brought into mechanical contact with the substrate (Fig. 1b) and experiments are carried out by sliding the probe laterally on the surface. To ensure bending of the array probe in a pre-defined direction after touching the sample, the soft probes are approached with an inclination angle $\gamma$ determined by the home-made holder system (Fig. 1c). Thanks to a screw and a rotatable plate (VI. in Fig. 1c and Fig. 1d), $\gamma$ can be manually adjusted. Our tests showed that $\gamma=20^{\circ}$ (respect to the surface normal) constitutes a good compromise between achievable current contrast and frictionless probe sliding [50]. Before starting experiments with soft probes, two important manual manipulation steps of critical importance must be carried out: $(i)$ cutting of the array to expose the cross-section containing the active microelectrode areas and (ii) placing the array into the holder. On the one hand, fresh microelectrode surfaces and a setup ready-to-use are achieved very quickly by cutting of the crosssection and placing the array in the holder, but on the other hand, this might cause minor microelectrodes-sample misalignments. This is described by an angle $\varphi$, which is the angle between the sample surface and the probe edge defined by the array cross-section containing the active electrode areas and the Parylene C-coated probe body facing the sample substrate (Fig. 1e). If $\varphi \neq 0^{\circ}$, the individual working distances $d_{k}$ increase linearly from one side of the array (smallest $d$ ) to the other side (largest $d$ ). A worm drive was integrated into the probe holder for a horizontal rotation of the array probe ( $V$. in Fig. $1 \mathrm{c}$ and $\mathrm{e}$ ). In this way $\varphi$ can be adjusted manually. This effect is demonstrated by three experimental approach curves towards an insulating substrate with different values for $\varphi$ using an array of eight microelectrodes (Fig. 2). Variations in the absolute currents are due to minor differences in size and geometry of the individual microelectrodes [50]. For a perfectly aligned array $\left(\varphi=0^{\circ}\right)$, meaning the complete length of the edge of the crosssection is in mechanical contact with the substrate, similar negative feedback was obtained for all microelectrodes (Fig. 2a). The measured currents decreased due to the hindered diffusion of the redox mediator as the probe sample distance decreased until the array probe got in mechanical contact with the substrate surface. Then, the probe started to slide on the surface and the measured currents of each microelectrode did not change due to a constant $d$. The measured currents $i_{\mathrm{T}, k}$ were normalized to give $I^{\prime}$ using Eq. (1),

$I^{\prime}=\frac{i_{\mathrm{T}, k}\left(h_{\mathrm{p}}=0 \mu \mathrm{m}\right)}{i_{\mathrm{T}, \infty, k}}$

where $i_{\mathrm{T}, k}$ at $h_{\mathrm{P}}=0 \mu \mathrm{m}$ (probe in contact with the sample) is divided by the bulk current $i_{\mathrm{T}, \infty, k}$. In this way, the individually measured currents during approaching can be compared and their decrease can be used as in indicator for $d_{k}$. The calculated values for $I^{\prime}$ range from 0.45 to 0.58 and are shown in Table 1. A mean value of $0.49( \pm 0.05)$ demonstrates that all measured currents for $h_{\mathrm{P}}=0 \mu \mathrm{m}$ decreased to about the half indicating that all individual microelectrodes of the array are uniformly separated from the substrate surface in contact regime.

As soon as the probe and the sample are not aligned $\left(\varphi=1^{\circ}\right.$, Fig. 2b), the probe-sample contact is made only by one edge of the probe (inset in Fig. 2b). Due to a certain stiffness of the PET sheet, the working distances are not decreased further. Already the first working electrode $(k=1)$ shows less negative feedback

Table 1

Calculated normalized currents $I^{\prime}$ at $h_{\mathrm{p}}=0 \mu \mathrm{m}$ over an insulating substrate.

\begin{tabular}{lllllllll}
\hline$k$ & 1 & 2 & 3 & 4 & 5 & 6 & 7 & 8 \\
\hline$I^{\prime}\left(\varphi=0^{\circ}\right)$ & 0.52 & 0.48 & 0.43 & 0.47 & 0.58 & 0.45 & 0.50 & 0.51 \\
$I^{\prime}\left(\varphi=1^{\circ}\right)$ & 0.74 & 0.76 & 0.80 & 0.82 & 0.86 & 0.85 & 0.88 & 0.90 \\
$I^{\prime}\left(\varphi=4.6^{\circ}\right)$ & 0.92 & 0.93 & 0.94 & 0.95 & 0.97 & 0.97 & 0.98 & 0.99 \\
\hline
\end{tabular}




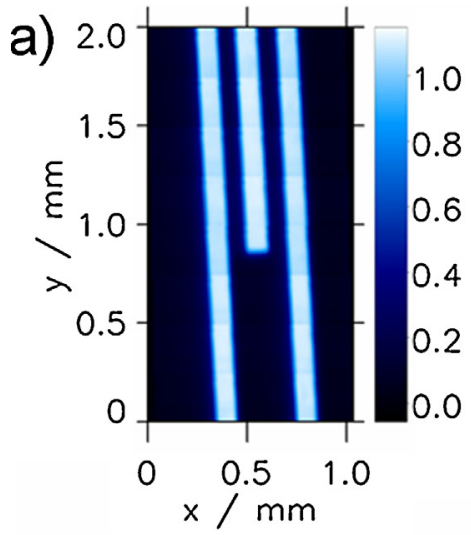

d)

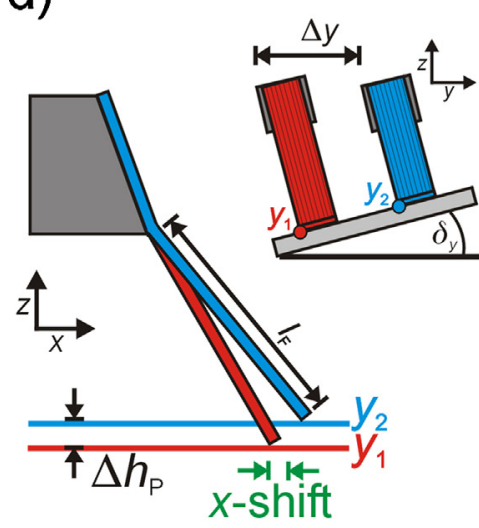

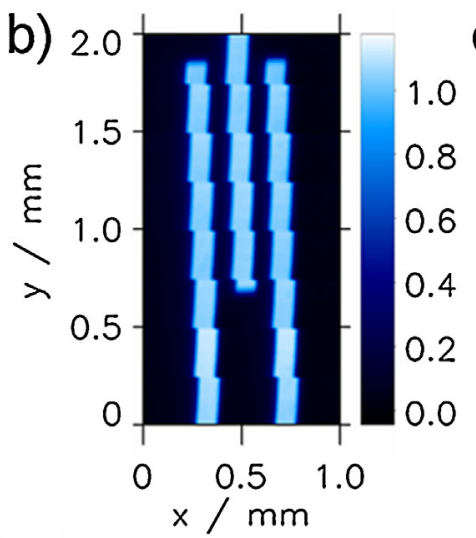

e)

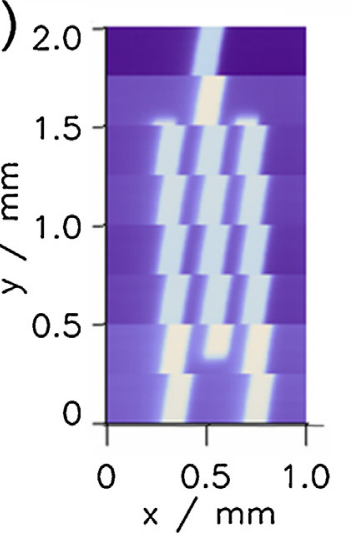

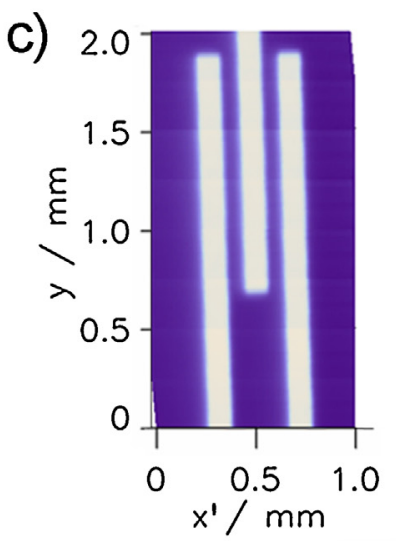

f)

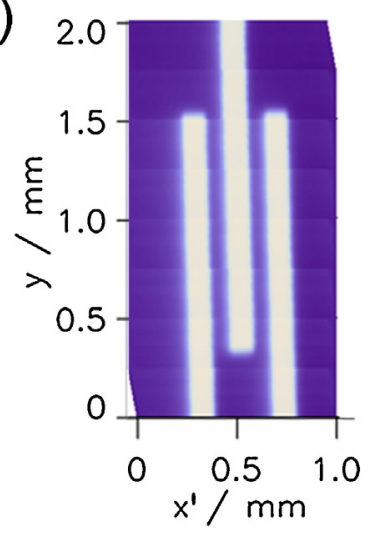

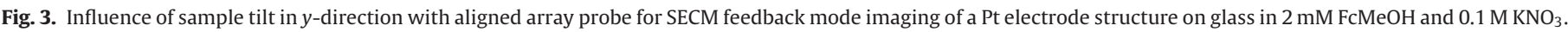

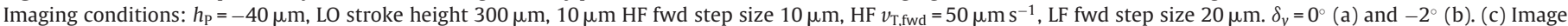

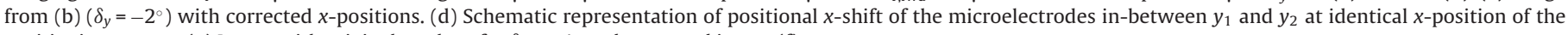
positioning system. (e) Image with original $x$ values for $\delta_{y}=-4^{\circ}$ and corrected image (f).

due to a less effective blocking of mediator diffusion to the active electrode $\left(I^{\prime}=0.74\right)$. From the first to the eighth microelectrode, $I^{\prime}$ increases linearly and shows a current reduction of only $10 \%$ for the 8th electrode. In principle, line scans and imaging can be performed under these conditions, but the resolution from $k=1$ to 8 will clearly decrease. This effect becomes even stronger for $\varphi=4.6^{\circ}$ (Fig. 2c) where the best condition $(k=1)$ provided only a $I^{\prime}$ of 0.92 and microelectrode 8 almost did not show any current decrease $\left(I^{\prime}=0.99\right)$. Although the array was approached until $h_{\mathrm{P}}=-90 \mu \mathrm{m}, d$ was not affected demonstrating that the soft probes are flexible enough allowing them to slide, but not to cling the whole probe on the surface. Therefore, a fast alignment of array and sample is recommended in order to achieve an optimum current contrast for feedback mode imaging. For this purpose, reflective substrate surfaces such as thin metallic films can be used for a manual array probe-sample alignment. The manual adjustment controlled by naked eye takes only a few seconds and $\varphi$ is usually significantly smaller than $1^{\circ}$ for reflecting metal surfaces where array and its mirror image are particularly conveniently used for alignment. A more precise alignment can then be achieved within several minutes by performing an approach curve and changing continuously $\varphi$.

\subsection{Sample tilt}

In general, the tilt of a plane sample can be described by two slopes, one in $x$-direction and one in $y$-direction. These are trigonometrically denoted as tilt angles $\delta_{x}$ and $\delta_{y}$, respectively, and can be controlled separately in our SECM setup by using the electronic tilt table. Fig. 3a shows the feedback mode image of a Pt microband structure on glass without surface tilt $\left(\delta_{x}=\delta_{y}=0^{\circ}\right)$. The tilt was eliminated prior to imaging and each individual microelectrode scanned an area of $1 \mathrm{~mm} \times 0.25 \mathrm{~mm}$, i.e. the array electrode covered an overall substrate region of $1 \mathrm{~mm} \times 2 \mathrm{~mm}$. Afterwards, the measured currents and positions of the individual microelectrodes of the array were corrected to give dimensionless currents $i_{\mathrm{T}}^{\prime} / i_{\mathrm{T} \text {, max }}$ $[49,53]$. The Pt structure is indicated in the SECM feedback image by high currents $\left(i^{\prime}{ }_{T} / i^{\prime}{ }_{T \text { max }} \approx 1.0\right)$ due to the regeneration of $\mathrm{FcMeOH}$ at the sample beneath the microelectrodes (positive feedback) while a calibrated current of zero is caused by the hindered diffusion of FcMeOH above the electronically insulating glass surface (negative feedback). The microelectrodes within the array performed exactly the same relative translations as the positioning system. Thus, the distances between sample regions e.g. the distances of the Pt electrodes can be estimated from the current profile of the reactivity image and match the real dimensions of the sample.

\subsubsection{Surface tilt in $y$-direction}

Tilting the sample in $y$-direction by about $\delta_{y}=-2^{\circ} \quad\left(\delta_{x}=0^{\circ}\right)$ requires a new probe-sample alignment. Note that such a large tilt is easily identifiable by naked eye (the variation of height is as large as $3.5 \mu \mathrm{m}$ for each $100 \mu \mathrm{m}$ of scan length) and would have been eliminated already before starting any measurement in conventional operation. Here an exaggerated tilt angle is used in order to illustrate more clearly the effects of surface tilts. An SECM feedback mode image (Fig. 3b) was recorded using the same parameters as in Fig. 3a. Due to the fact that the microelectrode arrays were aligned with respect to the substrate surface, the working distance $d$ was constant at each image point. However, the calibrated image shows clearly a continuous, simultaneous shift of the $x$-positions 
for each microelectrode in $y$-direction. Therefore, the eight individual images cannot construct an image representing the real surface dimensions of the Pt electrode pattern. The $x$-positions of microelectrode $k$ during the first line scan of the image are obviously different than the $x$-positions of microelectrode $(k-1)$ during the last line scan (which should be equal if the alignment is perfect as in Fig. 3a) resulting in a distorted image. This is due to the fact that the translation axes of the positioning system are neither parallel $(y)$ nor perpendicular $(z)$ to the substrate surface. During re-approaching after performing a step into the $y$-direction on a $y$-tilted sample, the probe will reach mechanical contact with the substrate surface at lower or higher absolute $z$-positions compared to the situation at the previous $y$-coordinate. This causes a change of the initial $h_{\mathrm{P}}$ value, denoted as $\Delta h_{\mathrm{P}}$, which depends on the step size $\Delta y$ performed by the $y$-axis of the positioning system and the sample tilt. The schematic representation showing all geometric arrangements can be found in Fig. A1 in Appendix A.1. The real step size $\Delta y^{\prime}$ on the sample surface is slightly larger than $\Delta y$ of the positioning system. For instance, the real step size $\Delta y^{\prime}$ taking into account a $20 \mu \mathrm{m}$ step $\Delta y$ was calculated for two different values for $\delta_{y}\left(-2^{\circ}\right.$ and $\left.-4^{\circ}\right)$ to be $20.01 \mu \mathrm{m}\left(\delta_{y}=-2^{\circ}\right)$ and $20.05 \mu \mathrm{m}\left(\delta_{y}=-4^{\circ}\right)$, respectively. These enhancements of 0.05 and $0.25 \%$ are negligible $\left(\Delta y^{\prime} \approx \Delta y\right)$ and were not taken into consideration in the following discussion. Changes of the $h_{\mathrm{P}}$ value are more important (Appendix A.1) and are estimated as

$\Delta h_{\mathrm{P}}=n \Delta y \tan \delta_{y}$

where $n$ is the incremental step number. A single step of $20 \mu \mathrm{m}$ in $y$-direction changes the $h_{\mathrm{P}}$ value by about $\Delta h_{\mathrm{P}}\left(-2^{\circ}\right)=-0.7 \mu \mathrm{m}$ (3.5\%) and $\Delta h_{\mathrm{P}}\left(-4^{\circ}\right)=-1.4 \mu \mathrm{m}(7 \%)$, respectively, and cannot be ignored. The changed $h_{\mathrm{p}}$ will lead to a sliding of the probe along the $x$-direction resulting in a variation of the tip position at each line scan. The straightforward geometric arrangements (Fig. A1b in Appendix A.1) can be used to calculate and to correct the shift of the $x$-positions (Fig. 3d).

$$
\begin{gathered}
x_{-40}-x_{-40+\Delta h_{\mathrm{P}}}=l_{\mathrm{F}}\left(\cos \left[\arcsin \frac{l_{\mathrm{F}} \cos \gamma+h_{\mathrm{P}}}{l_{\mathrm{F}}}\right]\right. \\
\left.-\cos \left[\arcsin \frac{l_{\mathrm{F}} \cos \gamma+h_{\mathrm{p}}-n \Delta y \tan \delta_{y}}{l_{\mathrm{F}}}\right]\right)
\end{gathered}
$$

$l_{\mathrm{F}}$ represents the length of the freely suspended part of the probe (Fig. 3d). A Taylor development of Eq. (3) where only the linear term was taken into consideration is given in Eq. (4) in order to express the $x$-shift with a simplified formula:

$$
x_{-40}-x_{-40+\Delta h_{\mathrm{P}}}=-\frac{\left(l_{\mathrm{F}} \cos \gamma+h_{\mathrm{P}}\right) n \Delta y \tan \delta_{y}}{l_{\mathrm{F}} \sqrt{1-\left(\frac{l_{\mathrm{F}} \cos \gamma+h_{\mathrm{p}}}{l_{\mathrm{F}}}\right)^{2}}}
$$

As follows from Eqs. (2) to (4), the shift of initial $x$-positions varies linearly with LF-steps as observed experimentally. This might lead to a noticeable distortion in the image due to the shift of the space coordinate in HF direction observed at all sensors with a skew angle $\varepsilon$ of

$\varepsilon=\tan \frac{x_{-40}-x_{-40+\Delta h_{\mathrm{P}}}}{n \Delta y}$

which is the tangents of the $x$-shift divided by the length of the $n$th step size in $y$-direction. This effect becomes more pronounced with the increase of sample tilt angles. Raw images in Fig. $3 \mathrm{~b}$ and e demonstrate the sample tilt effect at $\delta_{y}$ equal to $-2^{\circ}$ and $-4^{\circ}$, respectively, while Fig. $3 c$ and $f$ reveal the feedback colour maps with corrected positions. In this way, the individual images of the eight microelectrodes give one large image representing the real surface reactivity. Therefore, particular attention should be paid to
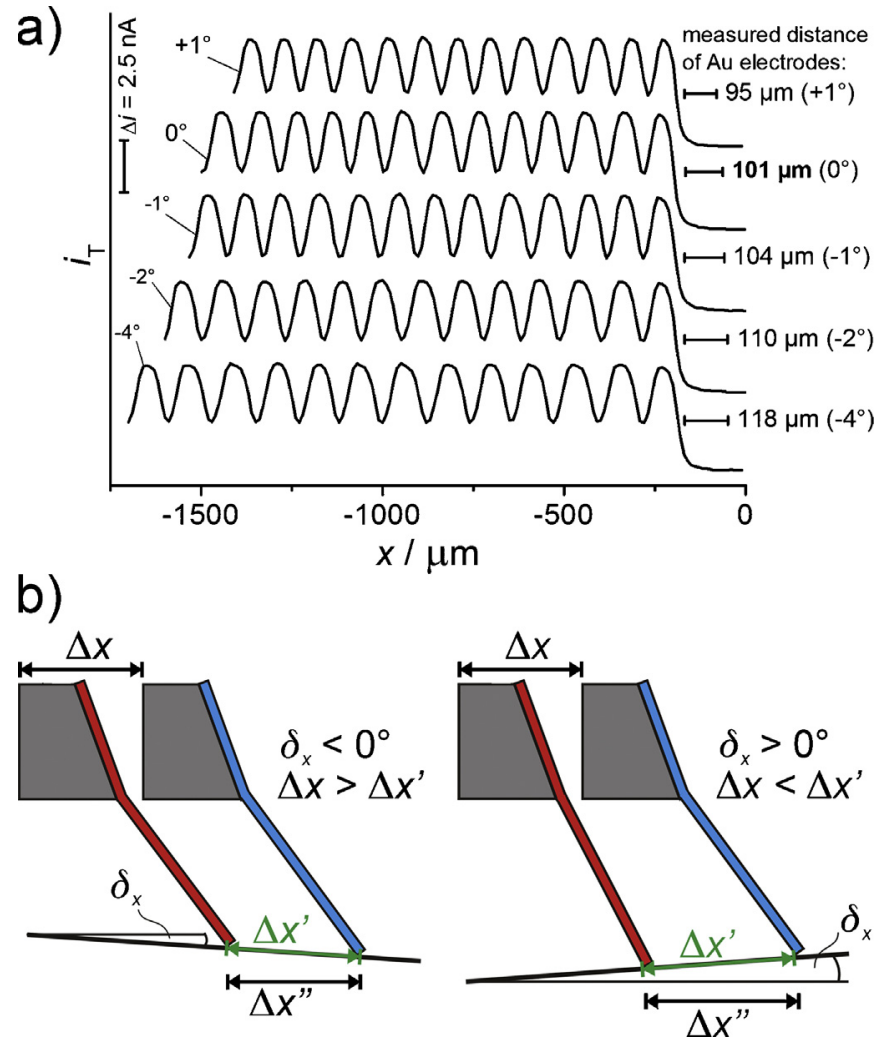

Fig. 4. (a) Feedback mode line scans of 13 gold electrodes on glass using one carbon microelectrode in a soft array. $\delta_{x}$ was $-1^{\circ}, 0^{\circ},+1^{\circ},+2^{\circ},+4^{\circ}$ which affects the real step size $\Delta x^{\prime}$. The gold bands appear compressed or stretched. (b) Schematic representation of the change of $\Delta x^{\prime}$ compared to the positioning system movements $\Delta x$ for $\delta_{x}<0^{\circ}$ and $\delta_{x}>0^{\circ}$.

the evaluation or elimination of tilt when scanning large surface areas prior to surface reactivity characterization.

\subsubsection{Surface tilt in $x$-direction}

A sample tilt in $x$-direction also affects the real $x$-step size significantly as shown in Fig. 4a. An interdigitated gold electrode array containing 13 tracks (midpoint-to-midpoint distance $100 \mu \mathrm{m}$ ) on glass was scanned applying several values of $\delta_{x}\left(-1^{\circ}\right.$ to $\left.+4^{\circ}\right)$ and using one microelectrode within a soft array probe. An average distance of the gold lines was calculated from the measured current profiles as a mean value of the 13 maximum currents over each line (i.e. approximately the middle of the width of the gold tracks). The measured value of $101 \mu \mathrm{m}$ for the horizontally aligned sample $\left(\delta_{x}=0^{\circ}\right)$ matches the real distance of the sample features. The gold lines appear to be narrower for $\delta_{x}>0^{\circ}$ and broader at $\delta_{x}<0^{\circ}$ leading to an image compression or extension, respectively. As follows from theory (Appendix A.2), the probe makes either larger or smaller real step sizes $\Delta x^{\prime}$ compared to $\Delta x$ of the positioning system and depending on $\delta_{x}$ (Fig. 4b). This fact is in a good agreement with experimental observations shown in Fig. 4a. For instance, variations of $\delta_{x}$ by $\pm 1^{\circ}$ changes the measured distance of the gold lines by $\pm 5 \mu \mathrm{m}$ (5\%) whereas theory predicts $4.3-4.7 \%$ variation.

The reported investigations made by applying defined sample tilt angles $\delta_{y}$ and $\delta_{x}$ demonstrate the behaviour of the sliding soft probes on strongly tilted, but plane substrates. In reality, tilts of flat samples are much smaller than the ones used herein and corrections for each measurement might not be required at all. The soft probes were developed for fast preparation and high performance of SECM experiments. Naturally, the tilt of a flat sample will be composed by both an $x$ - and $y$-component. Eqs. (6)-(21) in Appendices A. 1 and A.2 are valid for these cases as well. Furthermore, these 
a)
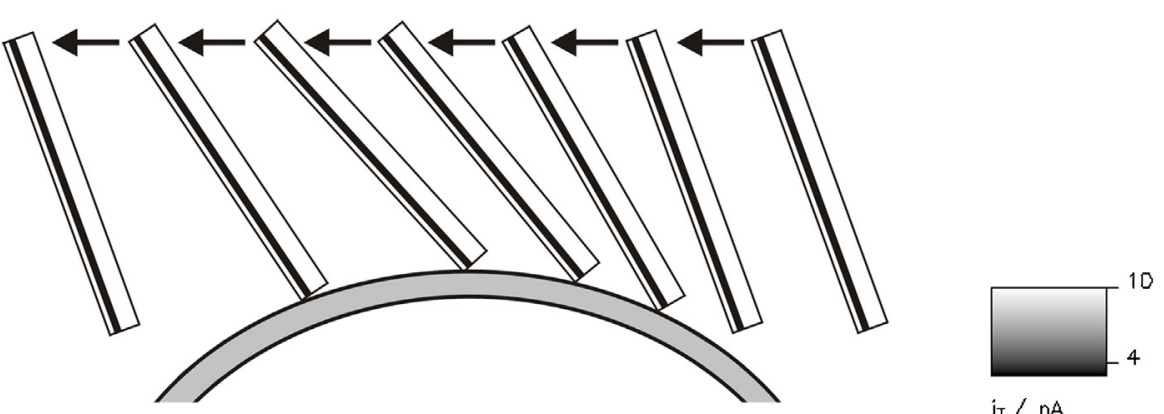

$\mathrm{i}_{\mathrm{T}} / \mathrm{nA}$

b)

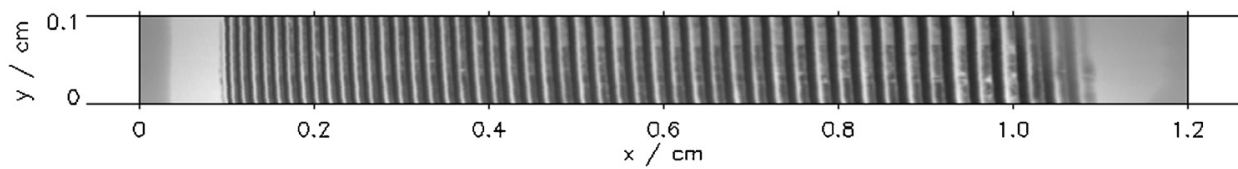

c)
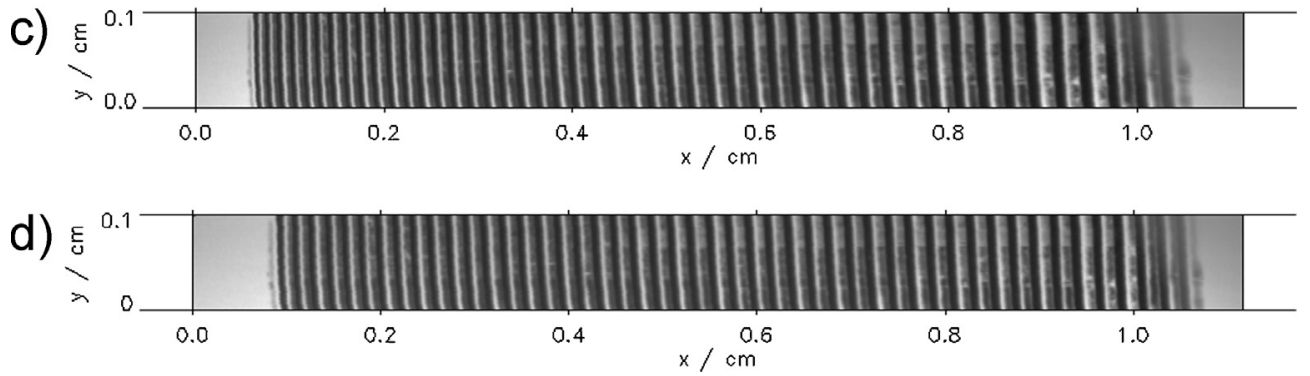

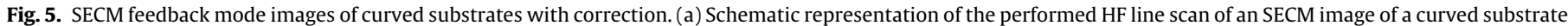

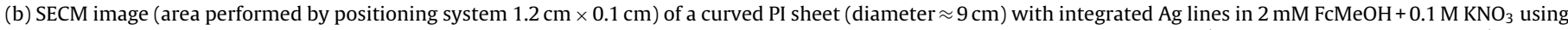

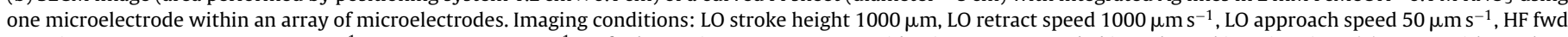

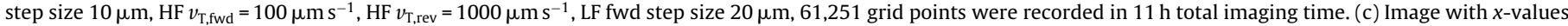
with mechanical probe-sample contact (56,814 data points). (d) Image with corrected $x$-positions (56,814 data points).

results illustrate how to interpret SECM images showing stretched or compressed surface structures. In order to measure the real extensions in such SECM images, the surface tilt could be eliminated as usually done when using conventional UMEs. Preferentially, different techniques such as SFM, confocal laser scanning microscopy (CLSM) or interferometric microscopy can be applied giving these dimensions and also topographic information with higher accuracy compared to SECM.

\subsection{Curved substrates}

An even more complex situation is found on curved and corrugated substrates where $\Delta x^{\prime}$ increase and/or decrease non-linearly within one line scan. This is due to the fact that $\delta_{x}$ varies at each point on the surface and causes an angular motion of the microelectrode arrays. In order to demonstrate this effect a curved substrate with integrated Ag silver lines was studied (Fig. 5a). Only minor efforts based on unaided optical inspection were made to eliminate sample tilt angles and to align the microelectrodes of the array with respect to the sample surface. Furthermore, the array was simply placed in close proximity to the curved sample, submerged in electrolyte solution, to allow contact mode scanning (Fig. 5a). A reactivity image of an area of $1.2 \mathrm{~cm}$ length and $0.1 \mathrm{~cm}$ width (travel distances of the positioning system) was scanned within $11 \mathrm{~h}$ with a point density of 5104 points $\mathrm{mm}^{-2}$ (10 $\mu \mathrm{m}$ steps; 61,251 data points; based on the scanned area of the positioning system) using one microelectrode of a soft array of eight carbon microelectrodes (Fig. 5b). At the beginning and at the end of each line scan of the scanning process, the probe was not in mechanical contact with the substrate as shown schematically in Fig. $5 \mathrm{a}$ and as indicated by the measured bulk currents for $x<370 \mu \mathrm{m}$ and $x>11,700 \mu \mathrm{m}$.
When the probe slides on the sample, a constant working distance is recorded along the entire image as indicated by the uniform current responses for either positive or negative feedback. During the first scanning part the increasing height of the curved substrate, which was $1.38 \mathrm{~mm}$ at the highest point, generates that the real step sizes become larger and the Ag lines appear narrower. At both edges of the measured image, the PI sample was covered with a homogenous layer of Ag. Similar to the corrections of the positional shifts in $x$-direction on flat tilted substrates, equations were developed to correct the lateral positions on the curved substrate (Appendix A.3). For this purpose, only data points for which the probe was in mechanical contact with the sample were taken into consideration. This results in an image of 56,814 data points and is shown in Fig. 5c. The average line thickness of the $\mathrm{Ag}$ lines in the original image is overestimated by about $32 \%$ at the right side of the image (start position of each line scan within an image) and underestimated by about $36 \%$ at the end of the scan (left side). In the corrected image (Fig. 5d) the widths of the Ag lines appear more uniform. However, there is still an overestimation of $8 \%$ at the right and an underestimation of $16 \%$ at the left side of the image. This can be explained by the practical difficulty to perfectly align the curved substrate to the $x$ - and $y$-axes of the positioning system and imperfections in estimating the exact curvature radius when fixing the flexible PI sample on the curved substrate. Therefore, the presented result shows the capability for fast imaging of unconventional tilted or curved samples with the soft probe arrays and their unambiguous interpretation in terms of the layout of the studied sample surfaces.

In order to demonstrate that a wide variety of large curved substrates can be scanned on a short time scale, an area of approximately $1.5 \mathrm{~cm} \times 0.8 \mathrm{~cm}$ of a pin from the Carl von Ossietzky University of Oldenburg (Fig. 6a) was scanned in SECM feedback 


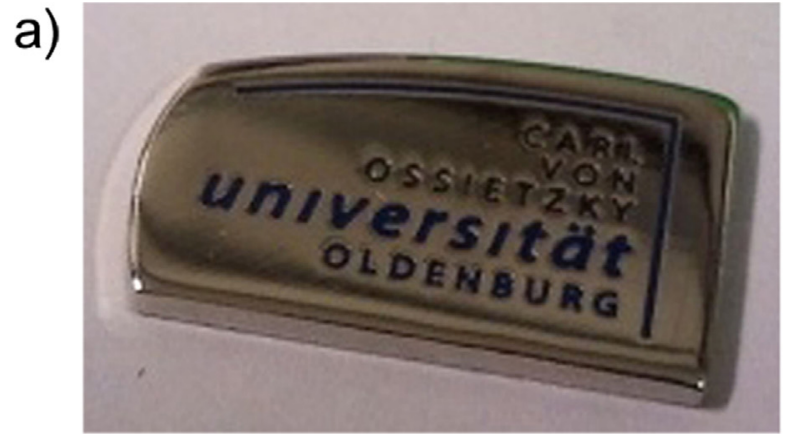

b)
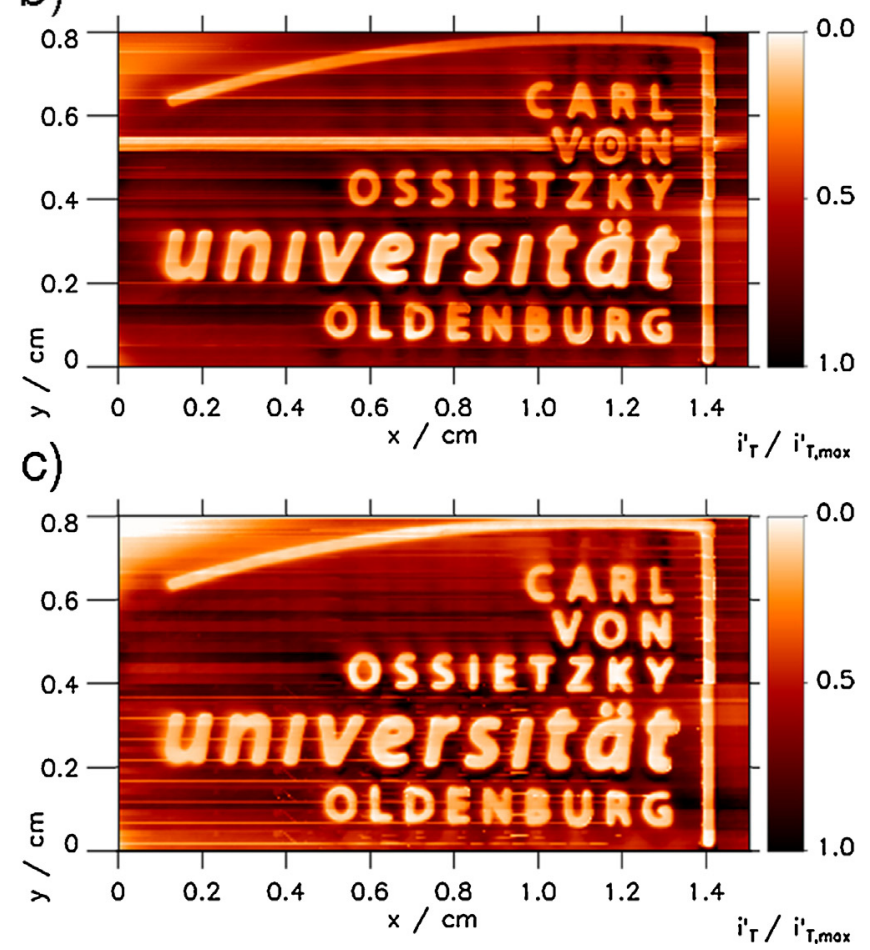

Fig. 6. SECM images of (a) a pin from the University of Oldenburg in $2 \mathrm{mM}$ $\mathrm{FcMeOH}+0.1 \mathrm{M} \mathrm{Na}_{2} \mathrm{SO}_{4}$. Imaging conditions: $h_{\mathrm{P}}=-100 \mu \mathrm{m}$, LO stroke height $500 \mu \mathrm{m}, \mathrm{HF} v_{\mathrm{T}, \mathrm{rev}}=1000 \mu \mathrm{m} \mathrm{s}^{-1}$, LF large step $4000 \mu \mathrm{m}$. (b) HF fwd step size $25 \mu \mathrm{m}$, $\mathrm{HF} v_{\mathrm{T}, \mathrm{fwd}}=50 \mu \mathrm{m} \mathrm{s}^{-1}$, LF fwd step size $25 \mu \mathrm{m}$, total imaging time required was $7 \mathrm{~h}$ $8 \mathrm{~min}$ for 192,320 grid points. (c) $\mathrm{HF}$ fwd step size $50 \mu \mathrm{m}$, HF $v_{\mathrm{T} \text { fwd }}=100 \mu \mathrm{m} \mathrm{s}^{-1}$, LF fwd step size $50 \mu \mathrm{m}, 1 \mathrm{~h} 55 \mathrm{~min}$ (48,160 data points).

mode using an array of eight carbon microelectrodes. The height profile measured along the $\operatorname{HF}(x)$ and $\operatorname{LF}(y)$ axes shows a curved shape and a rounding at the edges of the pin (Appendix A.4). The resulting image in Fig. $6 \mathrm{~b}$ is composed of two tiled images that are automatically recorded within one run by performing a large step of $4 \mathrm{~mm}$ in $y$ direction, when the first electrode starts to cover the area already scanned by the second electrode [49,50,53]. A relatively large step size of $25 \mu \mathrm{m}$ was sufficient with respect to the surface features. During an imaging time of $7 \mathrm{~h}, 192,320$ data points were obtained with a point density of 1603 points $\mathrm{mm}^{-2}$ (based on the scanned area of the positioning system). The metallic pin presents several letters engraved with various depths and filled by a lacquer. The soft array follows the curved topography of the pin but it cannot enter into the engraved characters because their dimensions are much smaller than the width of the array. The current responses rely on the positive feedback over the metallic surface and the current decrease over the engraved characters due to the increasing $d$, which results in the steady-state current in solution bulk. For constructing the image, this bulk current was normalized to zero. In Fig. 6b, an image frame from one electrode became damaged as indicated by the low currents $(y \approx 5.0-5.5 \mathrm{~mm}$ ). However, it could still detect the letters of the word "von". The resulting imaging time of $7 \mathrm{~h}$ is about 4.5 times less than the calculated time for a conventional SECM image using one UME with similar imaging parameters. In addition, a conventional SECM setup using a single classical UME cannot scan such a large curved area without additional instrumentation to control the working distance. For instance, $d$ would enlarge on the one side significantly or decrease on the other side, generating the possibility of a probesample crash. A feedback loop for distance control was recently applied in shear force-based SECM by Etienne et al. [45] who scanned a large bent steel substrate. Nine line scans of approx. $1.25 \mathrm{~mm}$ length were performed with a $25 \mu \mathrm{m}$ Pt-UME to measure over a distance of $8 \mathrm{~cm}$ perpendicular to the line scan direction. The probe followed the topography of the substrate and could go inside graves (about $45 \mu \mathrm{m}$ deep) but at the same time it showed some drawbacks. For instance, the step sizes (especially $1 \mathrm{~cm}$ along one horizontal axis) were larger than the UME diameter which led to an approximated point density of less than 4 points $\mathrm{mm}^{-2}$. Thus, many surface features are hardly detectable. Despite of this, the imaging time was still more than $4 \mathrm{~h}$ due to a finite time constant and the limited achievable scan rate of shear-force SECM [44].

In order to decrease the imaging time below $2 \mathrm{~h}$, both the translation rate and step size were doubled. With a point density of 401 points $\mathrm{mm}^{-2}$ (based on the movement of the positioning system; 48,160 grid points) a very good resolution (with respect to the sample features) was still achieved demonstrating the potential of soft probes for high-throughput imaging of curved and corrugated sample shapes (Fig. 6c). No correction procedures had to be performed. The dimensions are equal to those taken from optical microscopy and the engraved letters appear without distortion.

\section{Conclusions}

In this paper we demonstrated and proved that soft linear arrays enable SECM imaging of strongly tilted and curved substrates. The soft probes follow the topography of the sample surface in contact mode. This results in a constant working distance and thus in a uniform current for either positive or negative feedback situation. For a good current contrast, the individual microelectrodes of a soft array have to be placed as close as possible to the substrate. A minimum working distance for all microelectrodes of an array can be achieved by horizontal rotation of the electrode holder. Limitations arise for 3D-surface patterns when they are smaller than the width of the whole array. For instance, grooves of such size prohibit the array to go inside the surface structure and elevation force the whole array to lift off when passing over them. Both effects can increase the working distance for individual microelectrodes in a way that topographic features will be measured.

A misalignment between the horizontal axes of the positioning system and the surface of tilted or curved substrates causes lateral shifts in SECM images because the microelectrode probe bends differently in different regions. These shifts can be calculated and corrected as demonstrated for flat tilted and curved substrates.

By using soft linear microelectrode arrays, the dimensions of curved substrates that can be investigated on a very short time scale without making many efforts exceed the areas typically scanned by conventional SECM methodologies or other scanning probe techniques such as SFM by at least one order of magnitude. In this way, the soft arrays could be used to locate quickly defects of technical coatings, followed by a closer inspection with smaller electrodes.

\section{Acknowledgements}

The work was jointly supported by the Deutsche Forschungsgemeinschaft (DFG, Wi 1617/10) and the Fonds National Suisse pour la 
Recherche Scientifique (FNSRS, Grant No. 20PA21_121570/1) under the title "High throughput SECM imaging". The technical assistance by Valérie Devaud and Cyrille Hibert (both EPFL) is acknowledged, the authors also acknowledge Dr. Alexandr Shapeev for his kind help with Wolfram Mathematica code and computations. We thank Volker Steenhoff, NEXT ENERGY EWE-Forschungszentrum für Energietechnologie e.V., Oldenburg (Germany) for assistance in the profilometer measurement.

\section{Appendix A.}

\section{A.1. $y$-Tilt correction}

The real step size $\Delta y^{\prime}$ on the sample surface was calculated using Eq. (6). This phenomenon is shown schematically in Fig. A1a.

$\Delta y^{\prime}=\frac{\Delta y}{\cos \delta_{y}}$

Changes of the $h_{\mathrm{P}}$ value were calculated by Eq. (7).

$\Delta h_{\mathrm{P}}=n \Delta y \tan \delta_{y}$
Three situations are calculated and shown schematically in Fig. A1b: (i) the soft probe just in contact with the substrate surface $\left(h_{\mathrm{P}}=0 \mu \mathrm{m}\right.$; black triangle); (ii) the probe approached until $h_{\mathrm{P}}=-40 \mu \mathrm{m}$ before starting the image (red triangle); (iii) the probe approached after several line scans at a different $y$-position, but at the same $x$-position (blue triangle). The length $l_{\mathrm{F}}$ represents the freely suspended part of the probe. If the probe is just in contact with the sample $\left(h_{\mathrm{P}}=0 \mu \mathrm{m}\right)$ the vertical length $z_{0}$ can be calculated.

$z_{0}=l_{\mathrm{F}} \cos \gamma$

If the probe is approached further until $h_{\mathrm{P}}=-40 \mu \mathrm{m}$, it starts to slide on the surface and the distance $x_{-40}$ is given by Eq. (9).

$x_{-40}=l_{\mathrm{F}} \cos \beta_{-40}$ (10).

This equation requires $\beta_{-40}$ which can be calculated with Eq.

a)

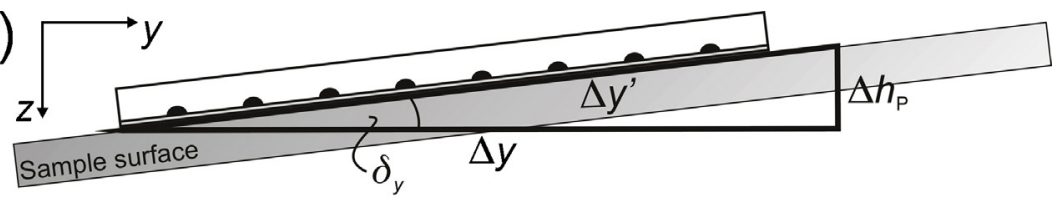

b)

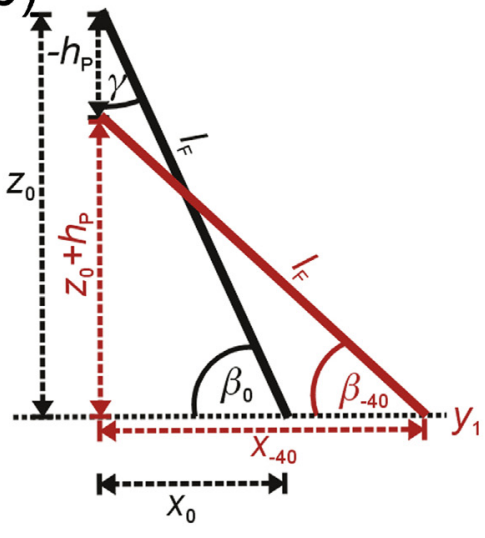

c)

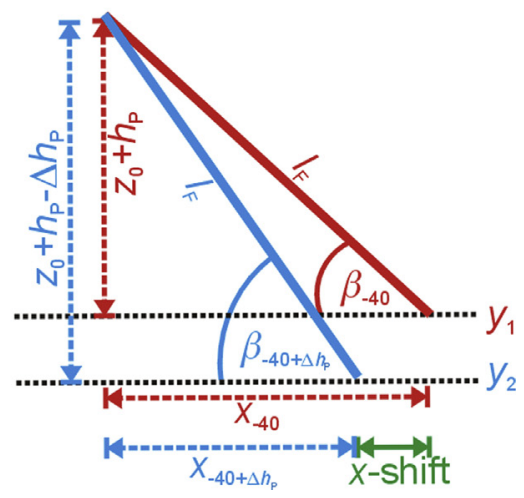

Fig. A1. Geometric arrangements of $\Delta y^{\prime}$ (a) and $x$-shift (b) with sample tilt in $y$-direction.

a

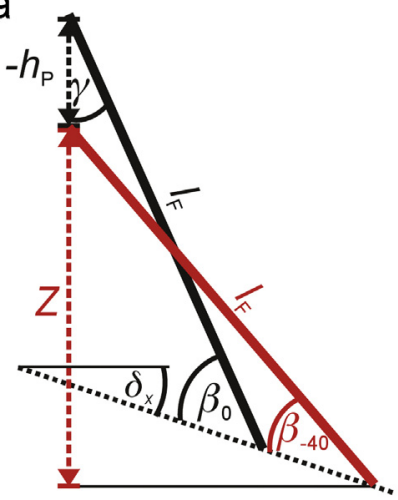

b

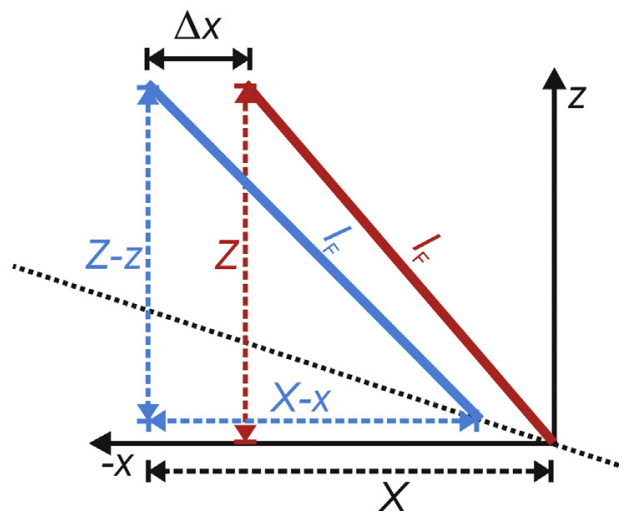

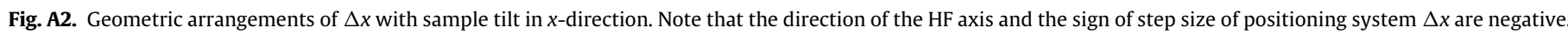



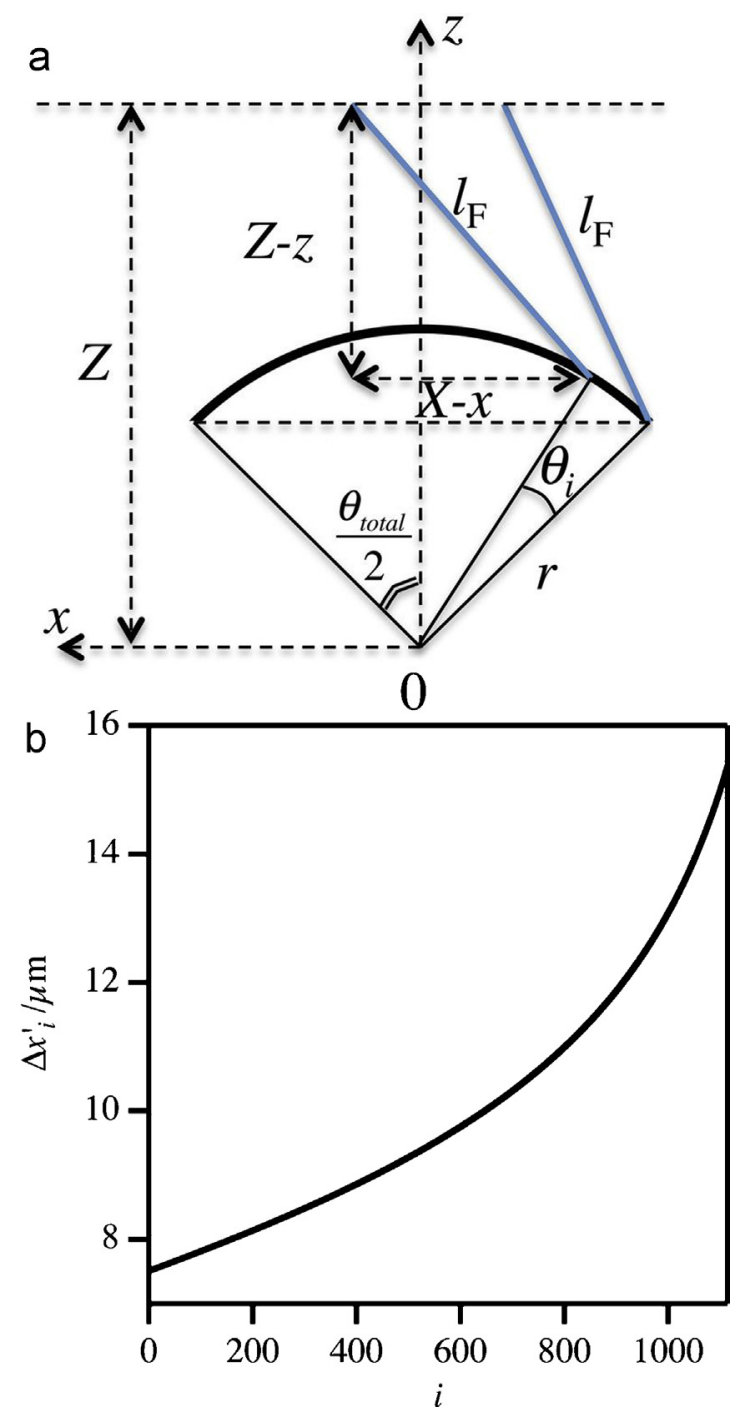

Fig. A3. Geometric arrangements to correct the probe movement (a) and non-linear increase of real step size $\Delta x^{\prime} v$ s. step number $n$ (b) on the curved substrate.

The value $x_{-40}$ represents the $x$ coordinate at starting position of the first line scan within an image. The subsequent $x$-positions of the probe apex in this line scan are calculated taking into account the shift of array initial position and overlay with the stored positions of the positioning system. The real starting $x$-positions of an array will change at each step in $y$-direction and can be calculated by

$x_{-40+\Delta h_{\mathrm{P}}}=l_{\mathrm{F}} \cos \beta_{-40+\Delta h_{\mathrm{P}}}$

while considering

$\beta_{-40+\Delta h_{\mathrm{P}}}=\arcsin \frac{z_{0}+h_{\mathrm{P}}-\Delta h_{\mathrm{P}}}{l_{\mathrm{F}}}=\arcsin \frac{l_{\mathrm{F}} \cos \gamma+h_{\mathrm{P}}-n \Delta y \tan \delta_{y}}{l_{\mathrm{F}}}$

\section{A.2. $x$-Tilt correction}

Fig. A2a shows the geometric arrangements for describing the effect of the sample tilt in $x$-direction on the real step size $\Delta x^{\prime}$. At the $n$th step in HF direction, the step size in the sample plane $\Delta x^{\prime}$ is

$\Delta x_{n}^{\prime}=\frac{x_{n}-x_{n-1}}{\cos \delta_{x}}$

where $x_{n}$ appears from the solution of equation system that describes linear movement of the microelectrode array tip

$z=x \tan \delta_{x}$

and the imaginary triangles having the hypotenuse $l_{\mathrm{F}}$ (as the probe length remains constant)

$(X-x)^{2}+(Z-z)^{2}=l_{\mathrm{F}}^{2}$

while $X$ and $Z$ are given by

$X=n \Delta x+l_{\mathrm{F}} \cos \alpha_{-40}$

$Z=l_{\mathrm{F}} \sin \alpha_{-40}$

where $\alpha_{-40}=\beta_{-40}+\delta_{x}$. The value of the angle $\alpha_{-40}$ at the beginning of each line scan is given by the solution of the Eq. (17) based on the properties of similar triangles and straightforward trigonometric expressions from the illustrative scheme in Fig. A2b.

$l_{\mathrm{F}} \sin \alpha_{0}-h_{\mathrm{P}}+l_{\mathrm{F}}\left(\cos \alpha_{-40}-\cos \alpha_{0}\right) \tan \delta_{x}=l_{\mathrm{F}} \sin \alpha_{-40}$

where $\alpha_{0}=\beta_{0}+\delta_{x}$.

\section{A.3. Curved substrate correction}

Fig. A3 shows schematically the geometric arrangements for evaluating the real step size $\Delta x^{\prime}$ on the curved substrate. The relationship between the step size imposed by the positioning system $\Delta x$ and the distance passed by the probe in the sample plane at the $n$th step $\Delta x_{n}^{\prime}$ is of primary importance. The latter quantity is the arc length $r \theta_{n}$, where $r$ and $\theta_{n}$ denote the curvature radius and central angle (in radians) at $n$th step, respectively. As shown on Fig. A3a, the angular step $\theta_{n}$ is given by

$\theta_{n}=\arccos \left(\frac{x_{n}}{r}\right)-\arccos \left(\frac{x_{n-1}}{r}\right)$
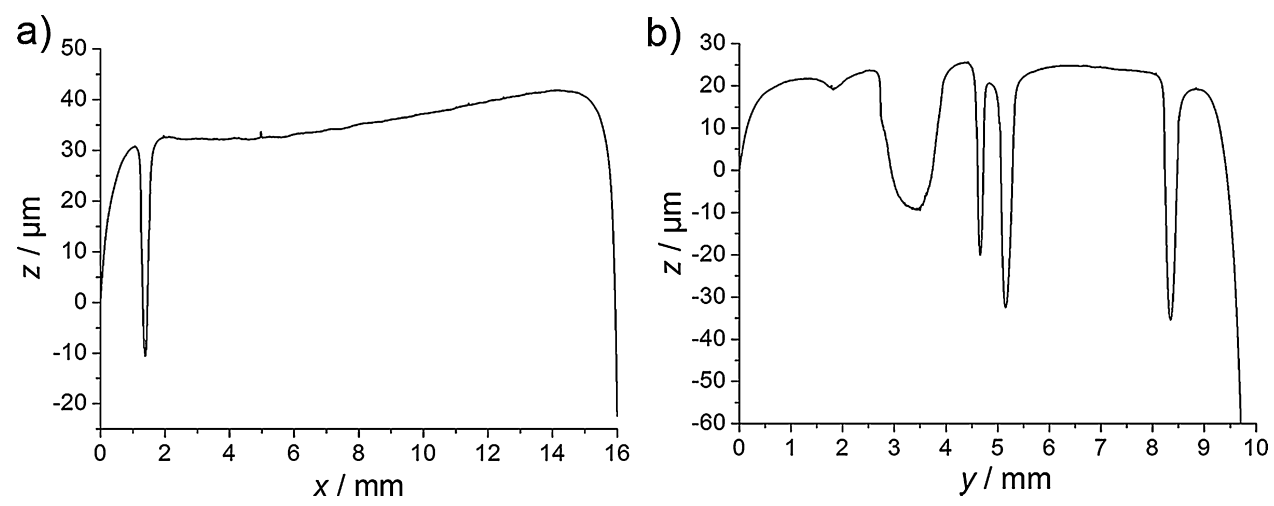

Fig. A4. Height profile of the test pin in scanned $x$ - (a) and $y$-direction (b). 
The argument $x_{n} \cdot r^{-1}$ could be found from the equation system that describes the angular motion and the imaginary triangle having the hypotenuse $l_{\mathrm{F}}$ (as the probe length remains constant).

$$
\left\{\begin{array}{c}
x^{2}+z^{2}=r^{2} \\
(X-x)^{2}+(Z-z)^{2}=l_{\mathrm{F}}^{2}
\end{array}\right.
$$

where $X$ and $Z$ are given by

$X=l_{\mathrm{F}} \cos \left(\frac{\pi}{2}-\gamma\right)-r \sin \left(\frac{\theta_{\text {total }}}{2}\right)+n \Delta x$

$Z=l_{\mathrm{F}} \sin \left(\frac{\pi}{2}-\gamma\right)+r \cos \left(\frac{\theta_{\text {total }}}{2}\right)$

and where $\theta_{\text {total }}$ represents the central angle of a curved substrate. The non-linear change of the real step size using the derived equations is shown in Fig. A3b. It demonstrates that $\Delta x^{\prime}$ increased continuously which can be seen in the SECM image as decreasing widths of the $\mathrm{Ag}$ lines.

\section{A.4. Test pin}

Fig. A4 shows the height profile of the pin from the University of Oldenburg along the $\operatorname{HF}(x)$ and $\operatorname{LF}(y)$ axes. A curved shape and a rounding especially at the edges of the pin were identified.

\section{References}

[1] R.C. Engstrom, M. Weber, D.J. Wunder, R. Burgess, S. Winquist, Measurements within the diffusion layer using a microelectrode probe, Analytical Chemistry 58 (1986) 844

[2] H.Y. Liu, F.R.F. Fan, C.W. Lin, A.J. Bard, Scanning electrochemical and tunneling ultramicroelectrode microscope for high-resolution examination of electrode surfaces in solution, Journal of the American Chemical Society 108 (1986) 3838

[3] A.J. Bard, F.R.F. Fan, D.T. Pierce, P.R. Unwin, D.O. Wipf, F.M. Zhou, Chemical imaging of surfaces with the scanning electrochemical microscope, Science 254 (1991) 68.

[4] G. Wittstock, M. Burchardt, S.E. Pust, Y. Shen, C. Zhao, Scanning electrochemical microscopy for direct imaging of reaction rates, Angewandte Chemie International Edition 46 (2007) 1584.

[5] D. Mandler, A.J. Bard, Scanning electrochemical microscopy - the application of the feedback mode for high-resolution copper etching, Journal of the Electrochemical Society 136 (1989) 3143.

[6] H. Shiku, T. Takeda, H. Yamada, T. Matsue, I. Uchida, Microfabrication and characterization of diaphorase-patterned surfaces by scanning electrochemical microscopy, Analytical Chemistry 67 (1995) 312.

[7] K. Borgwarth, C. Ricken, D.G. Ebling, J. Heinze, Surface characterization and modification by the scanning electrochemical microscope (SECM), Berichte der Bunsen-Gesellschaft für Physikalische Chemie 99 (1995) 1421.

[8] S. Nunige, R. Cornut, H. Hazimeh, F. Hauquier, C. Lefrou, C. Combellas, F. Kanoufi, Reactivity of surfaces determined by local electrochemical triggering: a bromo-terminated self-assembled monolayer, Angewandte Chemie International Edition 51 (2012) 5208.

[9] J.-M. Noel, A. Latus, C. Lagrost, E. Volanschi, P. Hapiot, Evidence for OH radical production during electrocatalysis of oxygen reduction on Pt surfaces: consequences and application, Journal of the American Chemical Society 134 (2012) 2835.

[10] C. Cougnon, F. Gohier, D. Belanger, J. Mauzeroll, In situ formation of diazonium salts from nitro precursors for scanning electrochemical microscopy patterning of surfaces, Angewandte Chemie International Edition 48 (2009) 4006.

[11] S. Rapino, G. Valenti, R. Marcu, M. Giorgio, M. Marcaccio, F. Paolucci, Microdrawing and highlighting a reactive surface, Journal of Materials Chemistry 20 (2010) 7272.

[12] G. Qin, M.Q. Zhang, T. Zhang, Y. Zhang, M. McIntosh, X. Li, X.J. Zhang, Label-free electrochemical imaging of latent fingerprints on metal surfaces, Electroanalysis 24 (2012) 1027.

[13] M. Zhang, H.H. Girault, SECM for imaging and detection of latent fingerprints, Analyst 134 (2009) 25

[14] J.L. Fernandez, D.A. Walsh, A.J. Bard, Thermodynamic guidelines for the design of bimetallic catalysts for oxygen electroreduction and rapid screening by scanning electrochemical microscopy. M-CO (M: Pd, Ag, Au), Journal of the American Chemical Society 127 (2005) 357.

[15] M. Black, J. Cooper, P. McGinn, Scanning electrochemical microscope characterization of thin film combinatorial libraries for fuel cell electrode applications, Measurement Science and Technology 16 (2005) 174.
[16] A. Minguzzi, M.A. Alpuche-Aviles, J.R. Lopez, S. Rondinini, A.J. Bard, Screening of oxygen evolution electrocatalysts by scanning electrochemical microscopy using a shielded tip approach, Analytical Chemistry 80 (2008) 4055.

[17] K. Fushimi, T. Okawa, K. Azumi, M. Seo, Heterogeneous growth of anodic oxide film on a polycrystalline titanium electrode observed with a scanning electrochemical microscope, Journal of the Electrochemical Society 147 (2000) 524.

[18] A. Maho, J. Denayer, J. Delhalle, Z. Mekhalif, Electro-assisted assembly of aliphatic thiol, dithiol and dithiocarboxylic acid monolayers on copper, Electrochimica Acta 56 (2011) 3954.

[19] R.M. Souto, Y. Gonzalez-Garcia, J. Izquierdo, S. Gonzalez, Examination of organic coatings on metallic substrates by scanning electrochemical microscopy in feedback mode: revealing the early stages of coating breakdown in corrosive environments, Corrosion Science 52 (2010) 748.

[20] T. Yasukawa, T. Kaya, T. Matsue, Dual imaging of topography and photosynthetic activity of a single protoplast by scanning electrochemical microscopy, Analytical Chemistry 71 (1999) 4637

[21] M.A. Alpuche-Aviles, D.O. Wipf, Impedance feedback control for scanning electrochemical microscopy, Analytical Chemistry 73 (2001) 4873.

[22] C. Gabrielli, F. Huet, M. Keddam, P. Rousseau, V. Vivier, Scanning electrochemical microscopy imaging by means of high-frequency impedance measurements in feedback mode, Journal of Physical Chemistry B 108 (2004) 11620.

[23] X.C. Zhao, P.M. Diakowski, Z.F. Ding, Deconvoluting topography and spatia physiological activity of live macrophage cells by scanning electrochemical microscopy in constant-distance mode, Analytical Chemistry 82 (2010) 8371.

[24] J.V. Macpherson, P.R. Unwin, Combined scanning electrochemical-atomic force microscopy, Analytical Chemistry 72 (2000) 276.

[25] C. Kranz, G. Friedbacher, B. Mizaikoff, Integrating an ultramicroelectrode in an AFM cantilever: combined technology for enhanced information, Analytical Chemistry 73 (2001) 2491

[26] A. Kueng, C. Kranz, A. Lugstein, E. Bertagnolli, B. Mizaikoff, Integrated AFMSECM in tapping mode: simultaneous topographical and electrochemical imaging of enzyme activity, Angewandte Chemie International Edition 42 (2003) 3238

[27] A. Davoodi, J. Pan, C. Leygraf, S. Norgren, Integrated AFM and SECM for in situ studies of localized corrosion of al alloys, Electrochimica Acta 52 (2007) 7697.

[28] S.E. Pust, M. Salomo, E. Oesterschulze, G. Wittstock, Influence of electrode size and geometry on electrochemical experiments with combined SECM-SFM probes, Nanotechnology 21 (2010) 105709.

[29] W. Smirnov, A. Kriele, R. Hoffmann, E. Sillero, J. Hees, O.A. Williams, N.J. Yang C. Kranz, C.E. Nebel, Diamond-modified AFM probes: from diamond nanowires to atomic force microscopy-integrated boron-doped diamond electrodes, Analytical Chemistry 83 (2011) 4936.

[30] A. Anne, A. Chovin, C. Demaille, M. Lafouresse, High-resolution mapping of redox-immunomarked proteins using electrochemical-atomic force microscopy in molecule touching mode, Analytical Chemistry 83 (2011) 7924.

[31] T.H. Treutler, G. Wittstock, Combination of an electrochemical tunneling microscope (ECSTM) and a scanning electrochemical microscope (SECM): application for tip-induced modification of self-assembled monolayers, Electrochimica Acta 48 (2003) 2923

[32] A.R. Kucernak, P.B. Chowdhury, C.P. Wilde, G.H. Kelsall, Y.Y. Zhu, D.E. Williams, Scanning electrochemical microscopy of a fuel-cell electrocatalyst deposited onto highly oriented pyrolytic graphite, Electrochimica Acta 45 (2000) 4483.

[33] D.J. Comstock, J.W. Elam, M.J. Pellin, M.C. Hersam, Integrated ultramicroelectrode-nanopipet probe for concurrent scanning electrochemical microscopy and scanning ion conductance microscopy, Analytical Chemistry 82 (2010) 1270 .

[34] Y. Takahashi, A.I. Shevchuk, P. Novak, Y. Murakami, H. Shiku, Y.E. Korchev, T. Matsue, Simultaneous noncontact topography and electrochemical imaging by SECM/SICM featuring ion current feedback regulation, Journal of the American Chemical Society 132 (2010) 10118.

[35] M.A. Edwards, A.L. Whitworth, P.R. Unwin, Quantitative analysis and application of tip position modulation-scanning electrochemical microscopy, Analytical Chemistry 83 (2011) 1977.

[36] D.O. Wipf, A.J. Bard, Scanning electrochemical microscopy. 15. Improvements in imaging via tip-position modulation and lock-in detection, Analytical Chemistry 64 (1992) 1362.

[37] D.O. Wipf, A.J. Bard, D.E. Tallman, Scanning electrochemical microscopy. 21 Constant-current imaging with an autoswitching controller, Analytical Chemistry 65 (1993) 1373.

[38] B. Ballesteros Katemann, A. Schulte, W. Schuhmann, Constant-distance mode scanning electrochemical microscopy (SECM) - Part I: Adaptation of a nonoptical shear-force-based positioning mode for SECM tips, Chemistry - A European Journal 9 (2003) 2025.

[39] M. Ludwig, C. Kranz, W. Schuhmann, H.E. Gaub, Topography feedback mechanism for the scanning electrochemical microscope based on hydrodynamic-forces between tip and sample, Review of Scientific Instruments 66 (1995) 2857

[40] D. Oyamatsu, Y. Hirano, N. Kanaya, Y. Mase, M. Nishizawa, T. Matsue, Imaging of enzyme activity by scanning electrochemical microscope equipped with feedback control for substrate-probe distance, Bioelectrochemistry 60 (2003) 115 . 
[41] Y. Lee, Z.F. Ding, A.J. Bard, Combined scanning electrochemical/optical microscopy with shear force and current feedback, Analytical Chemistry 74 (2002) 3634.

[42] P.I. James, L.F. Garfias-Mesias, P.J. Moyer, W.H. Smyrl, Scanning electrochemical microscopy with simultaneous independent topography, Journal of the Electrochemical Society 145 (1998) L64.

[43] M.F. Garay, J. Ufheil, K. Borgwarth, J. Heinze, Retrospective chemical analysis of tree rings by means of the scanning electrochemical microscopy with shear force feedback, Physical Chemistry Chemical Physics 6 (2004) 4028.

[44] U.M. Tefashe, G. Wittstock, Quantitative characterization of shear force regulation for scanning electrochemical microscopy, Comptes Rendus Chimie 16 (2012) 7.

[45] M. Etienne, B. Layoussifi, T. Giornelli, D. Jacquet, SECM-based automate equipped with a shearforce detection for the characterization of large and complex samples, Electrochemistry Communications 15 (2012) 70

[46] C. Dincer, E. Laubender, J. Hees, C.E. Nebel, G. Urban, J. Heinze, SECM detection of single boron doped diamond nanodes and nanoelectrode arrays using phaseoperated shear force technique, Electrochemistry Communications 24 (2012) 123

[47] K. McKelvey, M.A. Edwards, P.R. Unwin, Intermittent contact-scanning electrochemical microscopy (IC-SECM): A new approach for tip positioning and simultaneous imaging of interfacial topography and activity, Analytical Chemistry 82 (2010) 6334.

[48] Y. Takahashi, A.I. Shevchuk, P. Novak, B. Babakinejad, J. Macpherson, P.R. Unwin, H. Shiku, J. Gorelik, D. Klenerman, Y.E. Korchev, T. Matsue, Topographical and electrochemical nanoscale imaging of living cells using voltage-switching mode scanning electrochemical microscopy, Proceedings of the Nationa Academy of Sciences 109 (2012) 11540.

[49] A. Lesch, D. Momotenko, F. Cortes-Salazar, I. Wirth, U.M. Tefashe, F. Meiners, B. Vaske, H.H. Girault, G. Wittstock, Fabrication of soft gold microelectrode arrays as probes for scanning electrochemical microscopy, Journal of Electroanalytical Chemistry 666 (2012) 52.
[50] F. Cortes-Salazar, D. Momotenko, A. Lesch, G. Wittstock, H.H. Girault, Soft microelectrode linear array for scanning electrochemical microscopy, Analytical Chemistry 82 (2010) 10037.

[51] F. Cortes-Salazar, M. Träuble, F. Li, J.M. Busnel, A.L. Gassner, M. Hojeij, G. Wittstock, H.H. Girault, Soft stylus probes for scanning electrochemical microscopy, Analytical Chemistry 81 (2009) 6889.

[52] D. Momotenko, F. Cortes-Salazar, A. Lesch, G. Wittstock, H.H. Girault, Microfluidic push-pull probe for scanning electrochemical microscopy, Analytical Chemistry 83 (2011) 5275.

[53] A. Lesch, B. Vaske, F. Meiners, D. Momotenko, F. Cortes-Salazar, H.H. Girault, G. Wittstock, Parallel imaging and template-free patterning of self-assembled monolayers with soft linear microelectrode arrays, Angewandte Chemie International Edition 51 (2012) 10413.

[54] C. Combellas, M. Fermigier, A. Fuchs, F. Kanoufi, Scanning electrochemical microscopy. Hydrodynamics generated by the motion of a scanning tip and its consequences on the tip current, Analytical Chemistry 77 (2005) 7966.

[55] F. Cortes-Salazar, D. Momotenko, H.H. Girault, A. Lesch, G. Wittstock, Seeing big with scanning electrochemical microscopy, Analytical Chemistry 83 (2011) 1493.

[56] H. Meyer, H. Drewer, B. Gründig, K. Cammann, R. Kakerow, Y. Manioli, W. Mokwa, M. Rospert, Two-dimensional imaging of $\mathrm{O}_{2}, \mathrm{H}_{2} \mathrm{O}_{2}$, and glucose distributions by an array of 400 individually addressable microelectrodes, Analytical Chemistry 67 (1995) 1164.

[57] A.L. Barker, P.R. Unwin, J.W. Gardner, H. Rieley, A multi-electrode probe for parallel imaging in scanning electrochemical microscopy, Electrochemistry Communications 6 (2004) 91.

[58] F. Deiss, C. Combellas, C. Fretigny, N. Sojic, F. Kanoufi, Lithography by scanning electrochemical microscopy with a multiscaled electrode, Analytical Chemistry 82 (2010) 5169.

[59] K. Stulik, C. Amatore, K. Holub, V. Marecek, W. Kutner, Microelectrodes Definitions, characterization, and applications: (Technical report), Pure and Applied Chemistry 72 (2000) 1483. 